\title{
Giant cell arteritis associated with chronic active Epstein-Barr virus infection
}

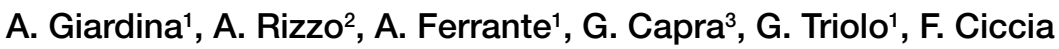 \\ 'Dipartimento Biomedico di Medicina Interna e Specialistica, Sezione di Reumatologia, \\ Università degli Studi di Palermo; \\ 2Unità Operativa di Anatomia Patologica, Ospedali Riuniti, Villa Sofia-Cervello, Palermo; § \\ ${ }^{3}$ Dipartimento di Scienze per la Promozione della Salute, Università degli Studi di Palermo, Italy
}

\begin{abstract}
SUMMARY
Giant cell arteritis is an inflammatory vasculopathy that preferentially affects medium-sized and large arteries. A viral cause has been suspected but not confirmed in polymyalgia rheumatica and giant-cell arteritis. We report the case of a 81-year-old female who suffered from chronic active Epstein-Barr virus infection and developed giant cell temporal arteritis.
\end{abstract}

Key words: Giant cell arteritis (GCA), Epstein Barr virus (EBV), chronic active EBV infection (CAEBV-infection).

Reumatismo, 2013; 65 (1): 36-39

\section{INTRODUCTION}

Gentan iant cell arteritis (GCA) is an inflammatory vasculopathy that preferentially affects medium- and large-sized arteries (1). An association with polymyalgia rheumatica occurs and both are probably polygenic diseases in which multiple environmental and genetic factors influence susceptibility and severity (1). Pathogenetic studies of GCA may suggest that GCA is an antigen driven disease (2). A viral cause has been suspected but not confirmed in polymyalgia rheumatica and giant-cell arteritis. The epidemiologic evidence that there is a similar cyclic fluctuation (every six to seven years) in the incidence of giant-cell arteritis and parvovirus B19 infection suggests in fact that viruses could have a role (3-5).

Chronic active Epstein-Barr virus infection (CAEBV) is characterized by infectious mononucleosis-like clinical features and elevated titres of EBV antibodies (6). Recently, EBV association with various etiologically unknown diseases has been demonstrated through the documentation of the presence or increase of viral genomes in disease-related lesions (7).

To the knowledge of authors no association has been described between CAEBV and GCA. In the present report we describe the case of a patient in whom GCA occurred during the course of CAEBV suggesting that EBV infection may play a pathogenetic role in the development of arteritis.

\section{CASE REPORT}

The patient was a 81-year-old female who suffered from two months of fever, generalized malaise, cervical lymph node enlargement, and hepatosplenomegaly. Six weeks later she complained the onset of headache, located over the temporal area and consistent with GCA-type headache, and was admitted to Palermo University Hospital. The patient suffered also from pain during swallowing and jaw claudication. Paresthesia of the scalp, carotidynia, or visual disturbances were not present. Physical examination on admission revealed enlarged cervical lymph nodes and hepatosplenomegaly. The frontal and parietal branches of the superficial temporal arteries were thickened, nodular, tender, and erythematous. Laboratory data revealed increased ESR and CRP $(80 \mathrm{~mm} / \mathrm{hr}$ and $30 \mathrm{mg} / \mathrm{L}$, respectively), moderate anaemia 
(hemoglobin, $85 \mathrm{~g} / \mathrm{L}$ ), thrombocytopenia (platelets, $90 \times 10^{9} / \mathrm{L}$ ), and polyclonal gammopathy $(\mathrm{IgG}, 34.6 \mathrm{~g} / \mathrm{L} ; \mathrm{IgA}, 5.5 \mathrm{~g} / \mathrm{L}$; and $\operatorname{IgM}, 1.6 \mathrm{~g} / \mathrm{L})$. Elevated levels of AST (2.3, ukat/L), ALT (1.7, ukat/L), and LDH (6.2, ukat/L) were seen. Serological tests for herpes simplex virus, parvovirus B19, and cytomegalovirus were negative. EBV antibodies revealed extremely high $\mathrm{IgG}$ antibody titres against viral capsid antigen (VCA) $\operatorname{IgG}(\geq 5.120)$, and against early antigen DR (EADR) IgG $(\geq 640)$. Negative results for Epstein-Barr nuclear antigens (EBNA) was also observed. Peripheral blood was obtained from the patient on referral, and viral load was determined on peripheral blood mononuclear cells (PBMC) by real-time quantitative polymerase chain reaction (PCR) with primer sets yielding DNA fragments corresponding to the internal repeat 1 and EBNA-2 regions of the virus genome. $\mathrm{PBMC}$ were positive for EBV DNA with an EBV loads greater than $10^{2.5}$ copies/mg DNA. The amount of EBV DNA was calculated as the number of virus copies per microgram
PBMC. The EBV detection limit in this assay was approximately 10 copies/mg DNA for PBMC. The diagnosis of GCA was considered and 4 temporal arterial biopsy specimens were obtained. Histological examination, on Hematoxylin and Eosin stained paraffin embedded sections, showed a moderate inflammatory infiltrate mainly composed of lymphocytes and histiocytes. Multinucleated giant cells were also present into the arterial wall (Fig. 1). Diagnosis of GCA was made according to the ACR criteria (8). Detection of EBV DNA through PCR analysis was also performed on fresh-frozen specimen of the diseased temporal artery tissue. EBV genome was clearly detected in temporal artery biopsy sample with an EBV loads greater than $10^{2.5}$ copies/mg DNA.

\section{DIsCussion}

Giant cell arteritis (GCA) is a chronic inflammatory disorder with a strong predilection for elderly people. Although a

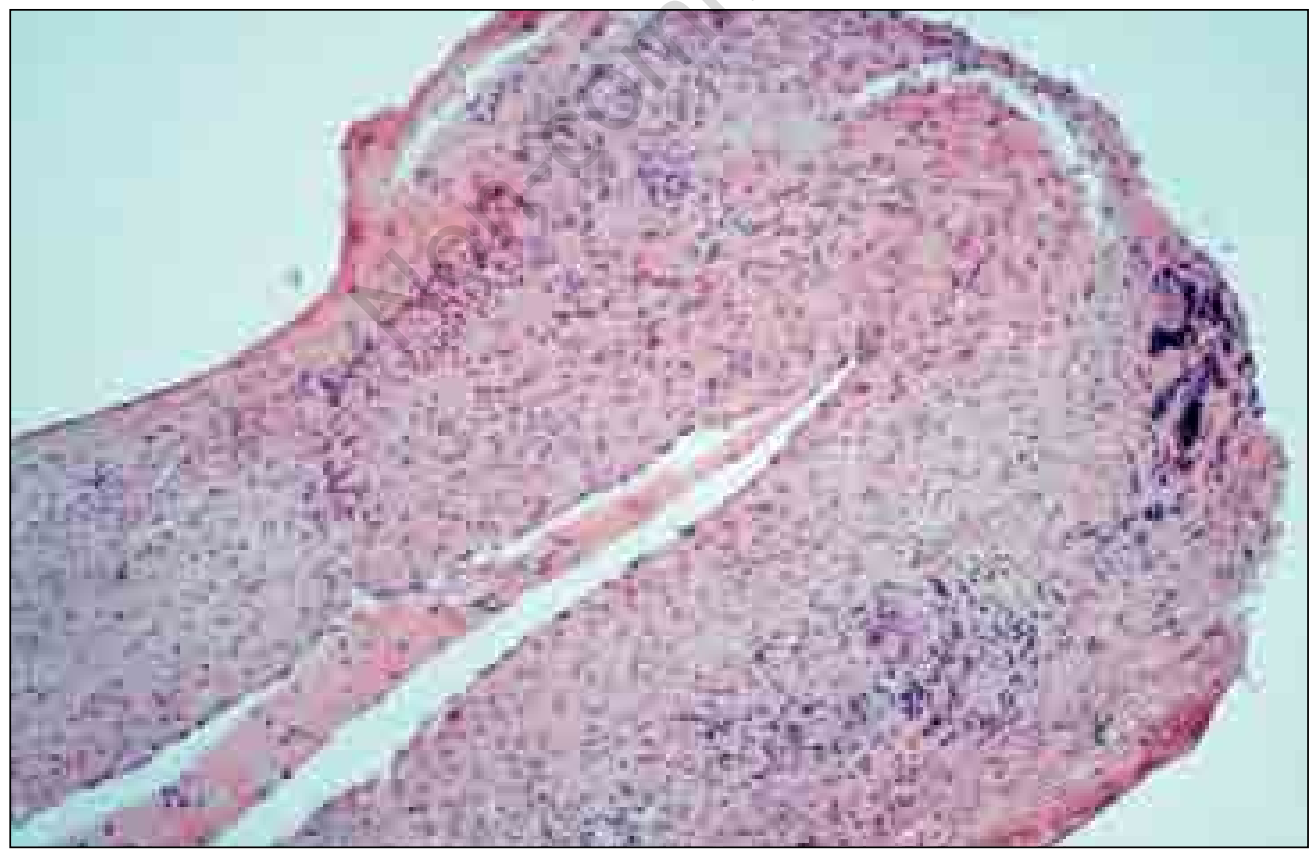

Figure 1 - Representative photomicrograph showing 5 - $\mu$ m-thick paraffin embedded sections of arterial biopsy specimens obtained from the patient stained with hematoxylin and eosin. Infiltration of T lymphocytes around and into the vasa vasorum together with the occurence of giant cells was evident in the arterial biopsy specimens obtained from the patient. 
correlation between the occurrence of infection and the onset of GCA has been demonstrated (9), suggesting that infection may act as a trigger mechanism in the pathogenesis of this syndrome, never a viral cause has been confirmed and the pathogenesis of this arteritis remains still unclear. In particular, the study from Cankovic and Zarbo (10) failed to confirm an association between HSV1, cytomegalovirus, and Epstein-Barr virus in 35 patients with histologically positive temporal arteries, apparently ruling out a viral pathogenesis of GCA.

Primary EBV infection usually occurs asymptomatically in early adulthood, but sometimes it results in infectious mononucleosis, which resolves spontaneously after the emergence of EBV-specific immunity (7). EBV may cause chronic infections in immunocompetent hosts. In healthy carriers, EBV exists latently in resting memory $B$ cells (7), whereas clonal expansion of EBV-infected T or NK cells is associated with chronic active EBV (CAEBV) infection (7), probably through the release of inflammatory cytokines such as interferon gamma, IL-6, or tumor necrosis factor alpha. This is the first report, to our knowledge, of the occurrence of GCA in a patient affected by CAEBV.

CAEBV infection is characterized by chronic or recurrent infectious mononucleosis-like symptoms and by an unusual pattern of anti-EBV antibodies (7). Hematologic examination often shows a tendency for pancytopenia and polyclonal gammopathy. CAEBV is a disease with a high mortality and high morbidity potentially with life-threatening complications, such as interstitial pneumonia, lymphoma, coronary artery aneurysms, and central nervous system involvement. One of the most common characteristics of CAEBV is elevated titres of EBV antibody (VCA IgG rl:1280, EADR positive or EBNA negative) (1lJ2). Proposed guidelines for diagnosing CAEBV are:

1. severe illness lasting more than 6 months that began as a primary EBV infection and that was associated with abnormal EBV antibody titres, antiviral capsid antigens (VCA) immunoglobu- lin $\mathrm{G}(\mathrm{IgG})$ at least 5120, anti-early antigens (EA) IgG at least 640, or anti-EB nuclear antigens (EBNA) less than 2;

2. histologic evidence of organ involvement such as interstitial pneumonia, hypoplasia of some bone marrow elements, vasculitis, lymphadenitis, persistent hepatitis or splenomegaly; and

3. increased quantities of EBV in affected tissues (7).

It can not be excluded that giant cell arteritis and EBV infection in our patient may have occurred together by chance alone, and it should be noted that the detection of EBV DNA in the temporal artery specimens does not per se necessarily imply that EBV triggered giant cell arteritis. Cases of an association between CAEBV and large-vessel arteritis, namely coronary arteritis have been however reported (11) and the case of CAEBV described herein was complicated by the concomitant presence of clinical and hystopathologic findings of GCA. The characteristic histopathologic features were the infiltration of lymphocytes and histiocytes into the inflamed arterial wall, together with the occurrence of giant cells. Using PCR analysis we clearly demonstrated the EBV DNA in the blood and inflamed artery of the patients, suggesting that EBV-infection might have participated in the pathogenesis of the arteritis in this case.

A model for the pathogenesis of giant-cell arteritis has been proposed by Weyand and Goronzy (2). In the adventitia antigen driven $T$ cells may enter the artery through the vasa vasorum, undergo clonal expansion, and produce interferon gamma. Vasa vasorum vasculitis could be a primary moment in the pathogenesis of GCA as demonstrated by their production of cytokines of the early innate immune response, such as IL-32 (12), a recently described proinflammatory cytokine induced by several viruses $(13,14)$.

We hypothesize that an unusual EBV infection into $\mathrm{T}$ lymphocytes occurred in our patient and played a role in the pathogenesis of CAEBV-associated arteritis. Although the precise pathogenetic mechanisms underlying the progression to CAE- 
$\mathrm{BV}$ remain unclear, the vascular changes in this case may be suggestive of a CAEBVassociated disorder.

Conflict of interests: the authors declare no potential conflict of interests.

\section{REFERENCES}

1. Salvarani C, Cantini F, Hunder GG. Polymyalgia rheumatica and giant-cell arteritis. Lancet. 2008; 372: 234-45.

2. Weyand CM, Goronzy JJ. Arterial wall injury in giant cell arteritis. Arthritis Rheum. 1999; 42: 844-53.

3. Alvarez-Lafuente R, Fernandez-Gutierrez B, Jover JA, al. Human parvovirus B19, varicella zoster virus, and human herpes virus 6 in temporal artery biopsy specimens of patients with giant cell arteritis: analysis with quantitative real time polymerase chain reaction. Ann Rheum Dis. 2005; 64: 780-2.

4. Duhaut P, Bosshard S, Calvet A, et al. Giant cell arteritis, polymyalgia rheumatica, and viral hypotheses: a multicenter, prospective case-control study. Groupe de Recherche sur l'Arterite a Cellules Geantes. J Rheumatol. 1999; 26: 361-9.

5. Salvarani C, Farnetti E, Casali B, et al. Detection of parvovirus B19 DNA by polymerase chain reaction in giant cell arteritis: a casecontrol study. Arthritis Rheum. 2002; 46: 3099-101.

6. Rickinson AB. Chronic, symptomatic EpsteinBarr virus infection. Immunol Today. 1986; 7: 13-4.
7. Okano M, Kawa K, Kimura H, et al. Proposed guidelines for diagnosing chronic active Epstein-Barr virus infection. Am J Hematol. 2005; 80: 64-9.

8. Hunder GG, Bloch DA, Michel BA, et al. The American College of Rheumatology 1990 criteria for the classification of giant cell arteritis. Arthritis Rheum. 1990; 33: 1122-8.

9. Russo MG, Waxman J, Abdoh AA, Serebro LH. Correlation between infection and the onset of the giant cell (temporal) arteritis syndrome. A trigger mechanism? Arthritis Rheum. 1995; 38: 374-80.

10. Cankovic M, Zarbo RJ. Failure to detect human herpes simplex virus, cytomegalovirus, and Epstein-Barr virus viral genomes in giant cell arteritis biopsy specimens by real-time quantitative polymerase chain reaction. Cardiovasc Pathol. 2006; 15: 280-6.

11. Murakami K, Ohsawa M, Hu SX, et al. Large-vessel arteritis associated with chronic active Epstein-Barr virus infection. Arthritis Rheum. 1998; 41: 369-73.

12. Ciccia F, Alessandro R, Rizzo A, et al. Expression of IL-32 in the inflamed arteries of patients with Giant Cell Arteritis. Arthritis Rheum. 2011; 63: 2097-104.

13. Lee S, Kim JH, Kim H, et al. Activation of the interleukin-32 pro-inflammatory pathway in response to human papillomavirus infection and over-expression of interleukin-32 controls the expression of the human papillomavirus oncogene. Immunology. 2011; 132: 410-20.

14. Moschen AR, Fritz T, Clouston AD, et al. IL-32: A new proinflammatory cytokine involved in HCV-related liver inflammation and fibrosis. Hepatology. 2011; Epub ahead of print. 